\title{
A CONTINUOUS TIME ANALOGUE OF THE THEORY OF RECURRENT EVENTS
}

\author{
BY J. F. C. KINGMAN
}

Communicated by William Feller, December 6, 1962

The theory of recurrent events developed by Feller [2] finds one of its most important applications in the theory of discrete time Markov chains. The object of this note is to summarise a continuous time analogue of Feller's theory which can be applied in a similar way to continuous time Markov chains.

1. The following definition of a (discrete time) recurrent event is readily seen to be equivalent to that of Feller. A recurrent event on a probability space $(\Omega, Q, P)$ is a family $\varepsilon=\{E(n), n=1,2, \cdots\}$ of Q-measurable subsets of $\Omega$, with the property that, for all positive integers $n_{1}<n_{2}<\cdots<n_{k}$,

$$
\begin{aligned}
P\left\{E\left(n_{1}\right) E\left(n_{2}\right) \cdots E\left(n_{k}\right)\right\} & \\
\quad & =P\left\{E\left(n_{1}\right)\right\} P\left\{E\left(n_{2}-n_{1}\right) \cdots E\left(n_{k}-n_{1}\right)\right\} .
\end{aligned}
$$

It follows that the probability of any event determined by the $E(n)$ can be calculated from a knowledge of the numbers

$$
u_{n}=P\{E(n)\},
$$

and thus much of the interest in the theory of recurrent events is centered on the "renewal sequence" $\left\{u_{n}\right\}$. Let us write $R$ for the class of all renewal sequences.

Because the word "recurrent" has come to be used in a different sense in Markov chain theory, we shall avoid it, and use instead the term "regenerative" to describe the events to be considered here. Then the form of the definition (1) suggests the following continuous time analogue.

A regenerative event $\varepsilon$ on a probability space $(\Omega, a, P)$ is a family of $Q$-measurable subsets $E(t)(t>0)$ of $\Omega$, having the property that, whenever real numbers $t_{j}$ satisfy

$$
0<t_{1}<t_{2}<\cdots<t_{k}
$$

then

(4) $P\left\{E\left(t_{1}\right) E\left(t_{2}\right) \cdots E\left(t_{k}\right)\right\}=P\left\{E\left(t_{1}\right)\right\} P\left\{E\left(t_{2}-t_{1}\right) \cdots E\left(t_{k}-t_{1}\right)\right\}$.

The function $p(t)$ defined by

$$
p(t)=P\{E(t)\}
$$


will be called the p-function of the event $\varepsilon$. From (4) we have

$$
P\left\{\bigcap_{j=1}^{k} E\left(t_{j}\right)\right\}=\prod_{j=1}^{k} p\left(t_{j}-t_{j-1}\right),
$$

where $t_{0}=0$. Hence a knowledge of $p(t)$ determines the probability of any subset of $\Omega$ which belongs to the $\sigma$-algebra $\mathcal{F}$ generated by the sets $E(t)$. It follows that the study of the properties of the function $p(t)$ will play a large part in the theory of regenerative events.

2. The class of all $p$-functions is characterised by the following theorem.

THEOREM I. Let $p(t)$ be any real function of $t>0$, and write

$$
\begin{aligned}
\Phi\left(t_{1}, t_{2}, \cdots, t_{k}\right) & =1-\sum_{1 \leq j_{1} \leq k} p\left(t_{j_{1}}\right)+\sum_{1 \leq j_{1}<j_{2} \leq k} p\left(t_{j_{1}}\right) p\left(t_{j_{2}}-t_{j_{1}}\right)-\cdots \\
& +(-1)^{k} \sum_{1 \leq j_{1}<\cdots<j_{k} \leq k} p\left(t_{j_{1}}\right) p\left(t_{j_{2}}-t_{j_{1}}\right) \cdots p\left(t_{j_{k}}-t_{j_{k-1}}\right),
\end{aligned}
$$

whenever $t_{1}, \cdots, t_{k}$ satisfy (3). Then there exists a regenerative event $\varepsilon$ with p-function $p(t)$ if and only if, whenever $k \geqq 1$ and $t_{1}, \cdots, t_{k}$ satisfy (3), we have

$$
0 \leqq \Phi\left(t_{1}, \cdots, t_{k}\right) \leqq \Phi\left(t_{1}, \cdots, t_{k-1}\right) .
$$

In particular, every $p$-function satisfies the inequalities

$$
p(t) p(u) \leqq p(t+u) \leqq 1+p(t) p(u)-\max \{p(t), p(u)\} .
$$

Let us say that $\varepsilon$ is standard if $p(t) \rightarrow 1$ as $t \rightarrow 0$, and let us denote by $\beta$ the class of all $p$-functions of standard regenerative events. Then the inequalities (7) imply a considerable regularity of analytic behavior for functions in $P$.

TheOREM II. If $p(t)$ belongs to $P$, then $p(t)$ is strictly positive and uniformly continuous in $0<t<\infty$. Moreover, $p(t)$ is of bounded variation in $0<t<T$ for every finite $T$, and is thus differentiable almost everywhere in $0<t<\infty$. The limit

$$
q=\lim _{t \rightarrow 0} t^{-1}\{1-p(t)\}
$$

exists (possibly infinite), and if $q<\infty$, then for all $t>0$,

$$
p(t) \geqq e^{-q t} \text {. }
$$

The proof of these results depends partly on the inequalities (7) and (8), and partly on the simple observation that, if $p(t) \in \mathcal{P}$, then (for any $h>0$ ) the sequence $\{p(n h)\}$ belongs to $R$. This remark also 
leads to the following characterisation of $P$.

THEOREM III. A continuous function $p(t)$ belongs to $\rho$ if and only if there exists a positive measure $\mu$ on $(0, \infty]$ satisfying

$$
\int\left(1-e^{-x}\right) \mu(d x)<\infty,
$$

and such that, for all $\theta>0$,

$$
r(\theta)=\int_{0}^{\infty} p(t) e^{-\theta t} d t=\left[\theta+\int_{(0, \infty]}\left(1-e^{-\theta x}\right) \mu(d x)\right]^{-1} .
$$

It follows from (12) that $p(t)$ satisfies the Volterra equation

$$
1-p(t)=\int_{0}^{t} p(t-s) \mu(s, \infty] d s .
$$

The Laplace transform in (12) can be inverted, and this leads to an expression for $p(t)$ in the form

$$
p(t)=\varpi+\int_{0}^{\infty} f(\lambda) \cos \lambda t d \lambda,
$$

where $\varpi \geqq 0$, and $f(\lambda)$ is non-negative and integrable. From this we deduce the following continuous time analogue of the Erdös-FellerPollard renewal theorem.

THEOREM IV. If $p(t) \in P$, then the limit

$$
\varpi=\lim _{t \rightarrow \infty} p(t)
$$

exists.

It should be remarked that this theorem neither implies nor is implied by the Blackwell renewal theorem, and in fact, the theory of regenerative events is distinct from continuous renewal theory.

3. Let $\varepsilon$ be a standard regenerative event, and define the stochastic process $Z(t, \omega)(t>0, \omega \in \Omega)$ by

$$
Z(t, \omega)=1 \text { (if } \omega \in E(t)), \quad Z(t, \omega)=0 \text { (otherwise). }
$$

Then $Z(t, \omega)$ is continuous in probability, and we may suppose (without essential loss of generality) that $Z$ is measurable and well-separable (in the sense of [1]). If this is done we may examine the random set

$$
S(\omega)=\{t ; t>0, \omega \in E(t)\} \text {. }
$$


It turns out that, if $q<\infty$, then $S(\omega)$ is (with probability one) made up of disjoint intervals, of which only finitely many meet any finite subinterval of the real line. If $q=\infty$, the structure of $S(\omega)$ is much more complex.

Define $\tau(t, \omega)$ to be the "time spent in $\mathcal{E}$ up to time $t$, " so that

$$
\tau(t, \omega)=\int_{0}^{t} Z(u, \omega) d u,
$$

and write $T(\tau, \omega)$ for the (left-continuous) inverse function of $\tau(t, \omega)$. Then $T(\tau, \omega)$ is a process with stationary independent increments, and its distributions are given by

$$
E\left\{e^{-\theta T(\tau, \omega)}\right\}=e^{-\tau / r(\theta)} .
$$

We say that $\mathcal{E}$ is transient if

$$
\bar{\tau}=\int_{0}^{\infty} p(t) d t<\infty .
$$

Then $\varepsilon$ is transient if and only if $\mu\{\infty\}>0$. If this is so, then $S(\omega)$ is bounded with probability one, and the Lebesgue measure $\tau(\omega)$ $=\tau(\infty, \omega)$ of $S(\omega)$ is exponentially distributed with mean $\bar{\tau}=1 / \mu\{\infty\}$. If $\mathcal{E}$ is not transient, then $\tau(\omega)$ is infinite with probability one.

4. Let $X(t, \omega)(t \geqq 0, \omega \in \Omega)$ be a continuous time Markov chain [1] with stationary transition probabilities $p_{i j}(t)(i, j=0,1,2, \cdots)$, and suppose that $X(0, \omega)=0$. Then the event $\varepsilon_{0}$ defined by

$$
E_{0}(t)=\{\omega ; X(t, \omega)=0\}
$$

is regenerative, with $p$-function $p_{00}(t)$. If the chain is standard, so is $\varepsilon_{0}$, and hence

$$
p_{00}(t) \in \boldsymbol{P} .
$$

Thus theorems about regenerative events imply immediately theorems about Markov chains.

It turns out that nearly all the known results relating to one state of a Markov chain are consequences of the fact that $\varepsilon_{0}$ is a regenerative event. An exception to this rule, however, is the theorem of Ornstein that $p_{00}(t)$ is differentiable in $t>0$; there are functions in $P$ which are not everywhere differentiable.

It can be shown that, if $p(t) \in \mathcal{P}$, then there exists a real-valued Markov process $X(t, \omega)$ such that

$$
p(t)=P\{X(t, \omega)=0 \mid X(0, \omega)=0\} .
$$


Thus any (standard) regenerative event can be represented by a Markov process on a continuous state space. It follows, for example, that the fact that $p_{00}(t)$ is almost everywhere differentiable is a consequence of the regenerative property of the state 0 , but the deeper result that $p_{00}(t)$ is everywhere differentiable requires also the discrete nature of the state space.

It is possible to extend the whole theory to take in properties of several states simultaneously, by considering systems of regenerative events. In particular, we can examine the transition probabilities $p_{i j}(t)(i \neq j)$ of a Markov chain. The theory may also be applied to certain Markov processes with continuous state space, and so, via the method of supplementary variables, to some non-Markovian processes.

It is hoped to publish elsewhere a detailed account of the theory summarised here, and of its various applications.

I am deeply grateful to Professor D. G. Kendall for much helpful discussion, and also to the Department of Scientific and Industrial Research for financial support.

\section{REFERENCES}

1. K. L. Chung, Markov chains with stationary transition probabilities, Springer, Berlin, 1960.

2. W. Feller, An introduction to probability theory and its applications, Wiley, New York, 1957.

University of Cambridge, Cambridge, England

\section{ORIENTABLE EMBEDDING OF CAYLEY GRAPHS}

BY WILLIAM GUSTIN

Communicated by J. W. T. Youngs, November 14, 1962

I present a method whereby a polygonal embedding of a Cayley graph in a closed oriented polyhedral surface may be represented as the dual of a quotient embedding of a quotient graph and diagrammed as a linked network of circuits carrying currents satisfying Kirchhoff's node law. By this means, devised to aid construction of triangular embeddings of a complete $n$ node to affirm Heawood's map color conjecture [3] in Heffter's dual formulation [4] for those cases $n \equiv 0,3,4,7 \bmod 12$ where such triangulation is compatible with Euler's polyhedral formula, I have been able to solve the cases $n \equiv 3,4,7 \bmod 12$, unaware that Ringel [5] had already resolved cases $n \equiv 3,7$ by a similar though less developed method. Case $n \equiv 0$ remains 